\title{
Fatores de risco e proteção para doenças crônicas não transmissíveis entre beneficiários da saúde suplementar: resultados do inquérito telefônico Vigitel, Brasil, 2008
}

\author{
Prevalence of risk health behavior \\ among members of private health insurance plans: \\ results from the 2008 national telephone survey Vigitel, Brazil
}

Deborah Carvalho Malta ${ }^{1}$

Martha Regina de Oliveira ${ }^{2}$

Erly Catarina de Moura ${ }^{3}$

Sara Araújo Silva ${ }^{1}$

Cláudia Soares Zouain ${ }^{2}$

Fausto Pereira dos Santos ${ }^{2}$

Otaliba Libanio de Morais Neto ${ }^{4}$

Gerson de Oliveira Penna ${ }^{4}$

${ }^{1}$ Coordenação Geral de Vigilância de Doenças e Agravos Não

Transmissíveis, Secretaria de Vigilância em Saúde, Ministério da Saúde. SAF Sul, trecho 02 , lotes 05 e 06 , bloco F, torre I, Edifício Premium, térreo, sala 14 70070-600 Brasília DF. deborah.malta@saude.gov.br

${ }^{2}$ Agência Nacional de Saúde Suplementar.

${ }^{3}$ Núcleo de Pesquisas em Nutrição, Universidade de São Paulo.

${ }^{4}$ Secretaria de Vigilância em Saúde, Ministério da Saúde.
Abstract This article aims at estimating the prevalence of adults engaging in protective and risk health behaviors among members of private health insurance plans. It was used a random sample of individuals over the age of 18 living in the Brazilian state capitals collected on 28,640 telephone interviews in 2008. The results showed that among males there was a high prevalence of the following risk factors: tobacco, overweight, low fruit and vegetable consumption, high meat with fat consumption and alcohol drinking. Among females we found a high prevalence of high blood pressure, diabetes, dyslipidemia and osteoporosis. Men were generally more physically active and women consumed more fruit and vegetables. As more educated males were lower was the prevalence of tobacco, high blood pressure, but also a higher prevalence of overweight, consumption of meat with fat, dyslipidemia and lower number of yearly checkups done. For females, tobacco smoking, overweight, obesity, decreasing with schooling, and consumption of fruit and vegetables, physical activity, mammography and PAP test, increased with schooling. The health insurance user population constitutes about $26 \%$ of Brazilian people and the current study aims to accumulate evidence for health promotion actions by this public.

Key words Chronic non-communicable diseases, Risk factor, Phone survey, Private health insurance
Resumo O objetivo deste artigo é descrever a distribuição dos principais fatores de risco (FR) e proteção para doenças crônicas não transmissíveis (DCNT) entre os beneficiários de planos de saúde. Foi utilizada amostra aleatória de adultos com 18 ou mais anos de idade nas capitais brasileiras, analisando-se frequências de FR em 28.640 indivíduos em 2008. Homens mostraram alta prevalência dos seguintes fatores de risco: tabaco, sobrepeso, baixo consumo de frutas e legumes, maior consumo de carnes gordurosas e álcool, enquanto mulheres mostraram maior prevalência de pressão arterial, diabetes, dislipidemia e osteoporose. Homens praticam mais atividade física e mulheres consomem mais frutas e vegetais. Homens com maior escolaridade apresentam maior frequência de sobrepeso, consumo de carnes com gorduras e dislipidemia. Entre mulheres, tabaco, sobrepeso, obesidade e doenças autorreferidas decrescem com aumento da escolaridade, enquanto o consumo de frutas e legumes, atividade física, mamografia e exame de papanicolau aumentam com a escolaridade. Conclusão: a população usuária de planos de saúde constitui cerca de $26 \%$ da população brasileira, e o estudo atual visa acumular evidências para atuação em ações de promoção da saúde para esse público.

Palavras-chave Doenças Crônicas Não Transmissiveis, Fatores de risco, Inquérito telefônico, Planos de saúde 


\section{Introdução}

Mais de $60 \%$ dos óbitos mundiais são devidos às doenças crônicas não transmissíveis (DCNT), segundo estimativas da Organização Mundial da Saúde (OMS) ${ }^{1}$. No Brasil, as DCNT constituem a principal causa de morte em adultos, com destaque para as doenças cardiovasculares, diabetes, neoplasias e doenças respiratórias crônicas, responsáveis pela maior parte das despesas com assistência ambulatorial e hospitalar ${ }^{2}$. O aumento da carga dessas doenças é consequência direta da urbanização acelerada, aumento progressivo da expectativa de vida, mudança no padrão alimentar, aumento do tabagismo e sedentarismo, entre outros fatores ${ }^{3,4}$. As DCNT também resultam em crescente impacto econômico, afetando famílias, sistema de saúde e sociedade ${ }^{1,4}$.

Estudos mostram que estratégias de controle dos fatores de risco dessas doenças, combinadas com educação comunitária e monitoramento dos indivíduos de alto risco, contribuíram para uma queda substancial na mortalidade em quase todos os países desenvolvidos ${ }^{5}$.

Considerando que as doenças crônicas não transmissíveis causam danos irreversíveis, apresentam um longo período assintomático e fatores de risco comuns e preveníveis, torna-se importante estabelecer um sistema de vigilância das DCNT que monitore seus fatores de risco e proteção, visando apoiar ações de promoção e prevenção $0^{2,4}$.

Nesse sentido, a Secretaria de Vigilância em Saúde implantou em 2006 a Vigilância de Fatores de Risco por Inquérito Telefônico (Vigitel), que se caracteriza por ser um sistema contínuo de monitoramento de fatores de risco e proteção das DCNT ${ }^{6,7}$. Em 2008, foi incluída uma questão sobre o uso de planos de saúde, possibilitando um olhar específico sobre a clientela beneficiária de planos de saúde.

Os beneficiários de planos de saúde no Brasil correspondem a cerca de $26 \%$ da população brasileira, ou seja, 49,2 milhões de pessoas, segundo a Pesquisa Nacional por Amostra de Domicílios (PNAD) 2008 ${ }^{8}$. Torna-se importante o monitoramento dessa população, e o trabalho atual reúne um conjunto de informações que podem apoiar a definição de práticas de promoção da saúde e prevenção de agravos e doenças direcionadas a essa população.

O objetivo deste estudo é descrever a distribuição dos principais fatores de risco e proteção para DCNT no conjunto das capitais brasileiras e do Distrito Federal no ano de 2008 para os beneficiários da saúde suplementar.

\section{Métodos}

São analisadas informações sobre fatores de risco (FR) e proteção extraídas do inquérito de fatores de risco e proteção para DCNT por entrevistas telefônicas (Vigitel) realizado em 2008 com a população adulta (com 18 anos de idade ou mais) residente em domicílios com pelo menos uma linha telefônica fixa nas 26 capitais de estados brasileiros e no Distrito Federal.

Foram realizadas, em 2008, 54.353 entrevistas, das quais se estimam em $41,8 \%$ a frequência de indivíduos na população adulta cobertos por planos de saúde em capitais, sendo 40,3\% com apenas um plano e 1,5\% com dois ou mais planos ${ }^{9}$. Para essa avaliação, foram utilizados os dados referentes à população beneficiária de planos de saúde, isto é, 28.640 indivíduos com 18 ou mais anos de idade.

Para estimar a frequência de cada indicador, foram utilizados fatores de ponderação construídos a partir da distribuição sociodemográfica (sexo, idade e escolaridade) do censo de 2000 para representar a população adulta total das cidades avaliadas. Foram construídas 24 categorias combinando: sexo (masculino ou feminino), faixa etária ( 18 a 34,35 a 44,45 a 54 ou $\geq 55$ anos de idade) e nível de escolaridade ( 0 a 8,9 a 11 ou $\geq 12$ anos de estudo). O peso final utilizado foi o resultado da multiplicação de três fatores: peso interno (número de adultos/número de linhas telefônicas de cada residência), razão censo-Vigitel (frequência de cada categoria no censo/frequência da mesma categoria na população beneficiária de planos de saúde por cidade) e fração amostral de cada cidade (número de habitantes adultos na cidade no censo de 2000/número de habitantes adultos beneficiários de planos de saúde avaliados pelo Vigitel na respectiva cidade). Mais informações sobre o desenho amostral e os procedimentos utilizados nas entrevistas encontram-se publicadas ${ }^{7,9}$.

Este estudo avalia as frequências de fatores de risco e proteção no conjunto das capitais brasileiras e do Distrito Federal. Dentre os fatores de risco, as seguintes variáveis foram avaliadas: fumantes (percentual de indivíduos que fumam, independentemente da frequência e intensidade do hábito de fumar); consumidores de vinte ou mais cigarros (percentual de indivíduos que fumam mais de vinte cigarros por dia); ex-fumantes (percentual de indivíduos que já fumaram em alguma época da vida); excesso de peso (percentual de indivíduos com índice de massa corporal igual ou superior a $25 \mathrm{~kg} / \mathrm{m}^{2}$ ); obesidade (percentual de indivíduos com índice de massa corporal igual ou 
superior a $30 \mathrm{~kg} / \mathrm{m}^{2}$ ); hábito de consumo de refrigerante (percentual de indivíduos que costumam consumir refrigerante ou suco artificial com açúcar cinco ou mais dias por semana) e de carnes com excesso de gordura (percentual de indivíduos que costumam consumir carne vermelha com gordura aparente ou frango sem a remoção da pele); consumo excessivo de bebidas alcoólicas (percentual de indivíduos que, nos últimos trinta dias, chegaram a consumir, em um único dia, mais de quatro (mulheres) ou cinco (homens) doses de bebida alcoólica); autoavaliação de estado de saúde ruim (percentual de indivíduos que autoavaliaram o estado de saúde como ruim ou muito ruim); referência a diagnóstico médico de hipertensão arterial (percentual de indivíduos que referem diagnóstico médico de hipertensão arterial), diabetes (percentual de indivíduos que referem diagnóstico médico de diabetes), dislipidemia (percentual de indivíduos que referem diagnóstico médico de dislipidemia); osteoporose (proporção de indivíduos que referem diagnóstico médico de osteoporose). Os fatores de proteção avaliados foram: hábito de consumo regular de frutas, legumes e verduras (FLV) (percentual de indivíduos que consomem FLV em cinco ou mais dias da semana) e de FLV conforme recomendado (percentual de indivíduos que consomem cinco ou mais porções diárias de FLV em cinco ou mais dias da semana); atividade física suficiente no tempo livre (lazer) (percentual de indivíduos que praticam atividades físicas de intensidade leve ou moderada por pelo menos trinta minutos diários em cinco ou mais dias por semana ou atividades de intensidade vigorosa por pelo menos vinte minutos diários em três ou mais dias da semana). Para as mulheres, avalia-se a realização de exames preventivos, como citologia oncótica nos últimos três anos para as mulheres entre $25 \mathrm{e}$ 59 anos de idade e mamografia nos últimos dois anos para as mulheres entre 50 e 69 anos de idade.

Para o processamento de dados e as análises estatísticas utilizou-se o aplicativo Stata versão 9.0, empregando-se comandos que computam proporções com intervalos de confiança que levam em conta o emprego de fatores de ponderação ${ }^{10}$.

Neste estudo, por se tratar de entrevista por telefone, o consentimento livre e esclarecido foi substituído pelo consentimento verbal obtido por ocasião dos contatos telefônicos com os entrevistados. Este estudo foi aprovado pela Comissão de Ética em Pesquisa em Seres Humanos do Ministério da Saúde.

\section{Resultados}

\section{Estimativas gerais}

A Tabela 1 ilustra as estimativas dos fatores de risco e proteção para DCNT em homens, mulheres e o total. Entre os beneficiários de planos de assistência à saúde, o hábito de fumar foi de $14,0 \%$, sendo maior em homens $(17,3 \%)$ do que em mulheres $(11,2 \%)$. A frequência de beneficiários de planos de saúde que declararam fumar vinte ou mais cigarros por dia foi de 3,9\%. A porcentagem de ex-fumantes foi de $21,0 \%$, sendo maior entre os homens $(25,0 \%)$ do que entre as mulheres $(17,5 \%)$. Os beneficiários de planos de saúde apresentaram um percentual de 46,9\% de excesso de peso e $14,1 \%$ de obesidade, sendo que o excesso de peso foi maior entre homens. No que se refere à alimentação, os beneficiários de planos de saúde referiram uma porcentagem de consumo regular de frutas, legumes e verduras de $36,1 \% ; 22,2 \%$ de consumo recomendado desses alimentos; $23,6 \%$ de consumo de refrigerantes; e $31,1 \%$ de consumo de carnes com gordura visível. À exceção do leite, as mulheres apresentam todos os demais comportamentos alimentares mais saudáveis do que os homens. A frequência de adultos que praticam atividade física suficiente no tempo livre foi maior no sexo masculino $(20,0 \%)$ do que no sexo feminino $(13,4 \%)$. No que tange ao consumo abusivo de bebidas alcoólicas, ou seja, mais de quatro doses para mulheres e mais de cinco para homens em uma mesma ocasião, nos últimos trinta dias, a frequência foi maior entre os homens $(26,8 \%)$ do que entre as mulheres $(9,7 \%)$. Com relação à avaliação do estado de saúde, um total de 3,8\% dos indivíduos estudados considerou seu estado de saúde como ruim e muito ruim, sendo maior entre as mulheres $(5,2 \%)$ do que entre os homens $(2,1 \%)$. Diagnósticos médicos prévios de hipertensão arterial, diabetes, dislipidemia e osteoporose foram referidos, respectivamente, por 23,4\%, 5,6\%, 19,0\% e 4,7\% dos beneficiários de planos de saúde. A frequência de mulheres entre 50 e 69 anos que realizaram exame de mamografia nos últimos dois anos foi de $83,6 \%$, e a frequência de mulheres entre 25 e 59 anos que realizaram o exame de citologia oncótica de colo de útero nos últimos três anos foi de $88,7 \%$.

\section{Faixas etárias}

As diferenças nas estimativas de fatores de risco e de proteção para DCNT quanto à faixa 
Tabela 1. Distribuição* (\%) de fatores de risco e proteção para doenças crônicas não transmissíveis em adultos ( $\geq 18$ anos) beneficiários de planos de saúde nas capitais brasileiras, segundo sexo, 2008.

\begin{tabular}{|c|c|c|c|c|c|c|}
\hline \multirow{3}{*}{ Indicadores } & \multirow{2}{*}{\multicolumn{2}{|c|}{ Total }} & \multicolumn{4}{|c|}{ Sexo } \\
\hline & & & \multicolumn{2}{|c|}{ Masculino } & \multicolumn{2}{|c|}{ Feminino } \\
\hline & $\%$ & (IC95\%) & $\%$ & (IC95\%) & $\%$ & (IC95\%) \\
\hline \multicolumn{7}{|l|}{ Hábito de fumar } \\
\hline Fumantes & 14,0 & $12,6-15,5$ & 17,3 & $14,7-19,9$ & 11,2 & $9,7-12,7$ \\
\hline Consumidores de vinte ou mais cigarros & 3,9 & $3,0-4,8$ & 5,3 & $3,6-7,0$ & 2,7 & $1,9-3,4$ \\
\hline Ex-fumantes & 21,0 & $19,5-22,4$ & 25,0 & $22,5-27,5$ & 17,5 & $15,8-19,3$ \\
\hline \multicolumn{7}{|l|}{ Estado nutricional } \\
\hline Excesso de peso & 46,9 & $44,9-48,8$ & 52,5 & $49,4-55,6$ & 41,7 & $39,4-44,1$ \\
\hline Obesidade & 14,1 & $12,7-15,5$ & 14,7 & $12,4-17,0$ & 13,5 & $11,9-15,2$ \\
\hline \multicolumn{7}{|l|}{ Hábito alimentar } \\
\hline FLV regular & 36,1 & $34,4-37,9$ & 29,4 & $26,7-32,0$ & 42,0 & $39,7-44,3$ \\
\hline FLV recomendado & 22,2 & $20,7-23,7$ & 17,1 & $15,1-19,1$ & 26,5 & $24,4-28,6$ \\
\hline Refrigerantes & 23,6 & $21,8-25,4$ & 27,4 & $24,5-30,2$ & 20,3 & $18,1-22,5$ \\
\hline Carnes com excesso de gordura & 31,1 & $29,2-33,0$ & 41,2 & $38,0-44,3$ & 22,5 & $20,4-24,5$ \\
\hline \multicolumn{7}{|l|}{ Atividade física } \\
\hline Ativo suficiente no tempo livre (lazer) & 16,4 & $15,0-17,9$ & 20,0 & $17,3-22,6$ & 13,4 & $12,1-14,8$ \\
\hline Consumo excessivo de álcool & 17,6 & $16,1-19,1$ & 26,8 & $24,1-29,5$ & 9,7 & $8,3-11,2$ \\
\hline Avaliação de estado de saúde ruim & 3,8 & $3,1-4,4$ & 2,1 & $1,4-2,7$ & 5,2 & $4,2-6,3$ \\
\hline \multicolumn{7}{|l|}{ Diagnóstico médico de doenças } \\
\hline Hipertensão arterial & 23,4 & $21,9-24,8$ & 21,2 & $19,0-23,4$ & 25,2 & $23,4-27,0$ \\
\hline Diabetes & 5,6 & $5,0-6,2$ & 4,9 & $4,0-5,8$ & 6,1 & $5,3-6,9$ \\
\hline Dislipidemia & 19,0 & $17,8-20,2$ & 15,3 & $13,6-17,1$ & 22,1 & $20,4-23,8$ \\
\hline Osteoporose & 4,7 & $4,2-5,2$ & 1,5 & $1,1-2,0$ & 7,5 & $6,6-8,3$ \\
\hline \multicolumn{7}{|l|}{ Exames preventivos } \\
\hline Mamografia $^{* *}$ & - & - & - & - & 83,6 & $81,0-86,2$ \\
\hline Citologia oncótica $^{x * x}$ & - & - & - & - & 88,7 & $86,7-90,7$ \\
\hline
\end{tabular}

* Percentual ponderado para ajustar a distribuição sociodemográfica da amostra Vigitel à distribuição da população adulta da cidade no censo demográfico de 2000; "* Exame realizado em mulheres de 50 a 69 anos nos últimos dois anos; "* Exame realizado em mulheres de 25 a 59 anos nos últimos três anos.

Fonte: Sistema de Vigilância de Fatores de Risco e Proteção para Doenças Crônicas por Inquérito Telefônico (Vigitel), Ministério da Saúde.

etária podem ser vistas nas tabelas 2 (homens) e 3 (mulheres). Em relação à distribuição por faixas etárias, observa-se que o hábito de fumar em homens é relativamente estável e em mulheres é menor entre 18 e 24 anos $(6,4 \%)$, crescendo até alcançar 17,2\% entre 45 e 54 anos e caindo para $4,2 \%$ após 65 anos de idade. O abandono do tabagismo entre homens e mulheres (ex-tabagistas) é maior a partir dos 45 anos de idade. O excesso de peso entre homens é maior nas faixas etárias mais altas, atingindo o ápice entre 45 e 54 anos $(67,3 \%)$. A obesidade segue o mesmo padrão. Entre mulheres, o excesso de peso também é maior nas faixas etárias mais altas, atingindo o ápice entre 55 e 64 anos (58,5\%). A obesidade também é maior nas faixas etárias mais altas. $\mathrm{O}$ consumo alimentar tende a ser melhor nas faixas etárias mais altas nos dois sexos. A atividade física entre os homens foi maior na faixa etária de 18 a 24 anos (31,2\%), e entre as mulheres não houve diferença. Em ambos os sexos, a frequência do consumo abusivo de bebidas alcoólicas foi maior nas faixas etárias mais jovens, caindo após os 55 anos de idade. A autoavaliação do estado de saúde ruim e muito ruim é maior nas faixas etárias mais altas. A referência ao diagnóstico de hipertensão arterial, diabetes, dislipidemia e osteoporose também é maior nas faixas etárias mais altas, tanto em homens quanto mulheres (tabelas 2 e 3 ).

\section{Escolaridade}

O hábito de fumar é maior entre os homens de menor escolaridade. $\mathrm{O}$ excesso de peso e a obesidade tendem a serem menores entre mu- 
Tabela 2. Distribuição* (\%) de fatores de risco e proteção para doenças crônicas não transmissíveis em homens beneficiários de planos de saúde nas capitais brasileiras, segundo faixa etária, 2008.

\begin{tabular}{|c|c|c|c|}
\hline \multirow[b]{2}{*}{ Indicadores } & \multicolumn{3}{|c|}{ Idade } \\
\hline & $\begin{array}{c}18 \text { a } 24 \\
\%(\text { IC } 95 \%)\end{array}$ & $\begin{array}{c}25 \text { a } 34 \\
\%(\text { IC95\%) }\end{array}$ & $\begin{array}{c}35 \text { a } 44 \\
\%(\text { IC95\%) }\end{array}$ \\
\hline \multicolumn{4}{|l|}{ Hábito de fumar } \\
\hline Fumantes & $18,4(11,5-25,3)$ & $18,0(11,5-24,6)$ & $17,2(12,9-21,4)$ \\
\hline Consumidores de vinte ou mais cigarros & $4,3(0,0-10,1)$ & $4,4(0,8-8,0)$ & $5,8(3,3-8,3)$ \\
\hline Ex-fumantes & $13,8(6,7-20,9)$ & $15,9(11,2-20,7)$ & $20,0(16,5-23,5)$ \\
\hline \multicolumn{4}{|l|}{ Estado nutricional } \\
\hline Excesso de peso & $28,2(22,0-34,4)$ & $53,1(45,5-60,7)$ & $60,6(55,7-65,5)$ \\
\hline Obesidade & $6,7(3,2-10,1)$ & $12,3(6,8-17,8)$ & $18,2(13,8-22,6)$ \\
\hline \multicolumn{4}{|l|}{ Hábito alimentar } \\
\hline FLV regular & $19,6(15,4-23,8)$ & $25,7(18,7-32,6)$ & $30,4(25,7-35,1)$ \\
\hline FLV recomendado & $12,2(8,9-15,6)$ & $14,5(9,0-19,9)$ & $18,6(14,8-22,4)$ \\
\hline Refrigerantes & $39,8(32,1-47,4)$ & $33,3(26,3-40,4)$ & $24,1(19,9-28,3)$ \\
\hline Carnes com excesso de gordura & $40,8(33,3-48,4)$ & $49,3(41,7-56,9)$ & $44,1(39,2-49,0)$ \\
\hline \multicolumn{4}{|l|}{ Atividade física } \\
\hline Ativo suficiente no tempo livre (lazer) & $31,2(23,9-38,4)$ & $18,4(11,7-25,1)$ & $13,8(10,2-17,3)$ \\
\hline Consumo excessivo de álcool & $27,3(21,3-33,3)$ & $31,8(24,9-38,8)$ & $29,6(25,2-34,1)$ \\
\hline Autoavaliação de estado de saúde ruim & $1,8(0,2-3,4)$ & $1,7(0,4-2,9)$ & $2,2(0,7-3,7)$ \\
\hline \multicolumn{4}{|l|}{ Diagnóstico médico de doenças } \\
\hline Hipertensão arterial & $5,3(2,7-8,0)$ & $9,9(5,6-14,2)$ & $18,7(15,1-22,4)$ \\
\hline Diabetes & $0,7(0,1-1,2)$ & $0,6(0,0-1,3)$ & $4,0(2,0-6,0)$ \\
\hline Dislipidemia & $3,8(2,5-5,2)$ & $5,8(4,2-7,3)$ & $19,6(15,9-23,3)$ \\
\hline \multirow[t]{2}{*}{ Osteoporose } & $0,2(0,0-0,4)$ & $0,3(0,0-0,8)$ & $1,4(0,0-2,8)$ \\
\hline & \multicolumn{3}{|c|}{ Idade } \\
\hline Indicadores & 45 a 54 & 55 a 64 & 65 ou mais \\
\hline & $\%($ IC95\%) & $\%($ IC95\%) & $\%($ IC95\%) \\
\hline \multicolumn{4}{|l|}{ Hábito de fumar } \\
\hline Fumantes & $19,7(15,1-24,3)$ & $14,4(10,2-18,5)$ & $10,9(6,3-15,4)$ \\
\hline Consumidores de vinte ou mais cigarros & $7,0(4,1-9,9)$ & $6,8(3,8-9,8)$ & $5,1(1,8-8,4)$ \\
\hline Ex-fumantes & $37,4(31,0-43,8)$ & $48,7(42,3-55,1)$ & $53,9(47,3-60,5)$ \\
\hline \multicolumn{4}{|l|}{ Estado nutricional } \\
\hline Excesso de peso & $67,3(61,5-73,1)$ & $64,7(58,4-71,0)$ & $52,5(45,8-59,2)$ \\
\hline Obesidade & $24,8(17,9-31,7)$ & $19,6(14,8-24,5)$ & $11,3(7,5-15,1)$ \\
\hline \multicolumn{4}{|l|}{ Hábito alimentar } \\
\hline FLV regular & $38,3(32,3-44,3)$ & $37,7(31,6-43,9)$ & $41,1(34,8-47,4)$ \\
\hline FLV recomendado & $19,4(15,4-23,4)$ & $21,9(17,1-26,7)$ & $26,3(20,4-32,2)$ \\
\hline Refrigerantes & $21,4(15,8-26,9)$ & $11,6(7,6-15,5)$ & $8,1(5,3-11,0)$ \\
\hline Carnes com excesso de gordura & $37,1(30,5-43,7)$ & $32,1(25,9-38,2)$ & $23,2(18,2-28,2)$ \\
\hline \multicolumn{4}{|l|}{ Atividade física } \\
\hline Ativo suficiente no tempo livre (lazer) & $15,8(11,9-19,7)$ & $17,9(12,8-23,1)$ & $20,7(15,8-25,5)$ \\
\hline Consumo excessivo de álcool & $30,7(24,1-37,3)$ & $14,7(11,1-18,2)$ & $6,2(4,0-8,3)$ \\
\hline Autoavaliação de estado de saúde ruim & $1,7(0,7-2,7)$ & $3,2(1,5-4,9)$ & $3,5(1,6-5,4)$ \\
\hline \multicolumn{4}{|l|}{ Diagnóstico médico de doenças } \\
\hline Hipertensão arterial & $40,0(33,3-46,8)$ & $48,8(42,4-55,2)$ & $49,3(42,7-55,8)$ \\
\hline Diabetes & $6,7(3,8-9,7)$ & $17,6(12,3-23,0)$ & $18,3(13,5-23,1)$ \\
\hline Dislipidemia & $30,8(24,3-37,3)$ & $30,9(25,2-36,5)$ & $24,9(19,1-30,8)$ \\
\hline Osteoporose & $1,6(0,5-2,6)$ & $3,2(0,9-5,6)$ & $8,0(5,0-11,0)$ \\
\hline
\end{tabular}

"Percentual ponderado para ajustar a distribuição sociodemográfica da amostra Vigitel à distribuição da população adulta da cidade no censo demográfico de 2000.

Fonte: Sistema de Vigilância de Fatores de Risco e Proteção para Doenças Crônicas por Inquérito Telefônico (Vigitel), Ministério da Saúde. 
Tabela 3. Distribuição* (\%) de fatores de risco e proteção para doenças crônicas não transmissíveis em mulheres beneficiárias de planos de saúde nas capitais brasileiras, segundo faixa etária, 2008.

\begin{tabular}{|c|c|c|c|}
\hline \multirow[b]{2}{*}{ Indicadores } & \multicolumn{3}{|c|}{ Idade } \\
\hline & $\begin{array}{c}18 \text { a } 24 \\
\%(\text { IC } 95 \%)\end{array}$ & $\begin{array}{c}25 \text { a } 34 \\
\%(\text { IC } 95 \%)\end{array}$ & $\begin{array}{c}35 \text { a } 44 \\
\%(\text { IC } 95 \%)\end{array}$ \\
\hline \multicolumn{4}{|l|}{ Hábito de fumar } \\
\hline Fumantes & $6,4(3,4-9,4)$ & $10,5(7,0-14,0)$ & $14,4(10,7-18,1)$ \\
\hline Consumidores de vinte ou mais cigarros & $1,1(0,1-2,1)$ & $1,3(0,3-2,3)$ & $4,4(1,9-6,9)$ \\
\hline Ex-fumantes & $8,6(5,3-12,0)$ & $13,2(8,5-17,9)$ & $16,3(13,6-18,9)$ \\
\hline \multicolumn{4}{|l|}{ Estado nutricional } \\
\hline Excesso de peso & $20,3(15,0-25,7)$ & $37,3(31,2-43,4)$ & $43,1(38,9-47,4)$ \\
\hline Obesidade & $3,4(1,1-5,7)$ & $11,6(7,2-15,9)$ & $13,8(10,8-16,8)$ \\
\hline \multicolumn{4}{|l|}{ Hábito alimentar } \\
\hline FLV regular & $28,5(23,4-33,5)$ & $38,8(32,7-44,9)$ & $42,5(38,4-46,6)$ \\
\hline FLV recomendado & $17,4(13,7-21,1)$ & $25,6(19,7-31,5)$ & $27,7(23,9-31,4)$ \\
\hline Refrigerantes & $31,9(26,2-37,7)$ & $29,7(23,8-35,6)$ & $16,3(13,1-19,5)$ \\
\hline Carnes com excesso de gordura & $26,3(21,2-31,5)$ & $28,8(23,2-34,4)$ & $21,5(18,1-24,9)$ \\
\hline \multicolumn{4}{|l|}{ Atividade física } \\
\hline Ativo suficiente no tempo livre (lazer) & $13,3(9,5-17,0)$ & $10,0(7,3-12,7)$ & $16,3(13,1-19,4)$ \\
\hline Consumo excessivo de álcool & $14,4(11,0-17,8)$ & $11,3(7,7-14,9)$ & $10,9(7,7-14,1)$ \\
\hline Autoavaliação de estado de saúde ruim & $4,7(1,1-8,2)$ & $3,3(1,2-5,4)$ & $4,2(2,4-6,0)$ \\
\hline \multicolumn{4}{|l|}{ Diagnóstico médico de doenças } \\
\hline Hipertensão arterial & $5,5(1,6-9,4)$ & $9,1(5,8-12,4)$ & $21,6(17,6-25,5)$ \\
\hline Diabetes & $0,8(0,3-1,4)$ & $0,5(0,2-0,9)$ & $3,3(1,5-5,2)$ \\
\hline Dislipidemia & $7,6(3,5-11,7)$ & $9,6(6,7-12,6)$ & $22,7(18,9-26,5)$ \\
\hline \multirow[t]{2}{*}{ Osteoporose } & $0,4(0,1-0,8)$ & $1,0(0,0-2,6)$ & $1,6(0,2-2,9)$ \\
\hline & \multicolumn{3}{|c|}{ Idade } \\
\hline Indicadores & 45 a 54 & 55 a 64 & 65 ou mais \\
\hline & $\%(\mathrm{IC} 95 \%)$ & $\%(\mathrm{IC} 95 \%)$ & $\%($ IC95\%) \\
\hline \multicolumn{4}{|l|}{ Hábito de fumar } \\
\hline Fumantes & $17,2(13,7-20,7)$ & $14,6(11,1-18,0)$ & $4,2(2,7-5,6)$ \\
\hline Consumidores de vinte ou mais cigarros & $5,5(3,3-7,8)$ & $3,6(2,0-5,2)$ & $0,8(0,4-1,3)$ \\
\hline Ex-fumantes & $29,0(25,2-32,9)$ & $27,6(23,6-31,5)$ & $22,5(19,4-25,7)$ \\
\hline \multicolumn{4}{|l|}{ Estado nutricional } \\
\hline Excesso de peso & $53,1(48,7-57,6)$ & $58,5(53,9-63,1)$ & $56,8(52,7-60,8)$ \\
\hline Obesidade & $18,3(14,6-22,1)$ & $21,6(17,5-25,8)$ & $21,6(18,1-25,1)$ \\
\hline \multicolumn{4}{|l|}{ Hábito alimentar } \\
\hline FLV regular & $47,8(43,5-52,1)$ & $53,3(48,8-57,8)$ & $54,0(50,2-57,9)$ \\
\hline FLV recomendado & $30,0(26,2-33,9)$ & $34,7(30,5-38,9)$ & $30,4(26,8-34,0)$ \\
\hline Refrigerantes & $10,8(8,5-13,2)$ & $9,6(6,6-12,5)$ & $6,5(4,7-8,2)$ \\
\hline Carnes com excesso de gordura & $18,2(15,0-21,4)$ & $18,9(15,0-22,8)$ & $10,5(8,2-12,9)$ \\
\hline \multicolumn{4}{|l|}{ Atividade física } \\
\hline Ativo suficiente no tempo livre (lazer) & $17,0(13,7-20,3)$ & $13,8(11,3-16,4)$ & $12,3(9,7-14,9)$ \\
\hline Consumo excessivo de álcool & $8,9(6,1-11,8)$ & $4,4(2,8-5,9)$ & $1,2(0,5-2,0)$ \\
\hline Autoavaliação de estado de saúde ruim & $6,2(3,7-8,7)$ & $5,9(4,0-7,8)$ & $10,9(8,4-13,4)$ \\
\hline \multicolumn{4}{|l|}{ Diagnóstico médico de doenças } \\
\hline Hipertensão arterial & $37,3(33,0-41,6)$ & $52,2(47,7-56,6)$ & $68,1(64,7-71,5)$ \\
\hline Diabetes & $9,6(6,5-12,6)$ & $13,9(10,5-17,2)$ & $23,6(20,1-27,1)$ \\
\hline Dislipidemia & $32,3(28,2-36,5)$ & $40,0(35,7-44,3)$ & $49,0(45,2-52,9)$ \\
\hline Osteoporose & $7,9(5,9-10,0)$ & $19,8(16,5-23,1)$ & $36,3(32,6-40,0)$ \\
\hline
\end{tabular}

* Percentual ponderado para ajustar a distribuição sociodemográfica da amostra Vigitel à distribuição da população adulta da cidade no censo demográfico de 2000.

Fonte: Sistema de Vigilância de Fatores de Risco e Proteção para Doenças Crônicas por Inquérito Telefônico (Vigitel), Ministério da Saúde. 
lheres com maior escolaridade. Comportamentos alimentares saudáveis melhoram nos níveis de maior escolaridade em ambos os sexos, exceto o consumo de refrigerantes entre homens, assim como a referência à atividade física suficiente no tempo livre. O consumo abusivo de bebidas alcoólicas não sofre influência da escolaridade. A autoavaliação de saúde ruim e muito ruim é maior em mulheres com menor escolaridade. A referência ao diagnóstico médico de doenças é menor entre mulheres com maior escolaridade; entre homens com maior escolaridade, é menor para diabetes, porém maior para dislipidemia. A frequência de realização de mamografia e de papanicolau é maior entre mulheres com maior escolaridade (tabelas 4 e 5).

\section{Discussão}

O estudo atual analisa os principais fatores de risco para DCNT na população usuária de pla- nos de saúde no Brasil. Outros estudos comparando os resultados aqui apresentados com a população não beneficiária de planos serão objeto de futuras publicações.

Segundo dados da Pesquisa Nacional por Amostra de Domicílios, a população coberta pelos planos de saúde cresceu de 24,5\% (2003) para $25,9 \%$ (2008), o que corresponde a um aumento de 43 milhões para 49,1 milhões de usuários em cinco anos ${ }^{8}$. Esta diferença é atribuída pelo IBGE ao crescimento econômico do país e consequente aumento do poder aquisitivo e emprego, já que o grande aumento dos planos ocorreu entre a população trabalhadora, afiliada aos planos coletivos, cofinanciados por empresas ${ }^{8}$. As regiões Sudeste e Sul registraram os maiores percentuais de cobertura $(35,6 \%$ e $30,0 \%$, respectivamente), aproximadamente três vezes maiores do que os verificados para o Norte $(13,3 \%)$ e o Nordeste (13,2\%). A cobertura por plano de saúde é maior na população de maior renda. Ape-

Tabela 4. Distribuição* (\%) de fatores de risco e proteção para doenças crônicas não transmissíveis em homens beneficiários de planos de saúde nas capitais brasileiras, segundo escolaridade, 2008.

\begin{tabular}{|c|c|c|c|c|c|c|}
\hline \multirow{3}{*}{ Indicadores } & \multicolumn{6}{|c|}{ Anos de estudo } \\
\hline & \multicolumn{2}{|r|}{0 a 8} & \multicolumn{2}{|c|}{9 a 11} & \multicolumn{2}{|c|}{12 ou mais } \\
\hline & $\%$ & (IC95\%) & $\%$ & $(\mathrm{IC} 95 \%)$ & $\%$ & (IC95\%) \\
\hline \multicolumn{7}{|l|}{ Hábito de fumar } \\
\hline Fumantes & 21,6 & $17,0-26,3$ & 12,4 & $10,6-14,3$ & 12,0 & $10,1-13,9$ \\
\hline Consumidores de vinte ou mais cigarros & 6,7 & $3,5-9,9$ & 3,5 & $2,6-4,5$ & 4,0 & $2,9-5,0$ \\
\hline Ex-fumantes & 28,7 & $24,1-33,2$ & 19,6 & $17,6-21,6$ & 22,5 & $20,2-24,7$ \\
\hline \multicolumn{7}{|l|}{ Estado nutricional } \\
\hline Excesso de peso & 51,5 & $45,9-57,1$ & 51,2 & $48,3-54,2$ & 57,5 & $54,7-60,3$ \\
\hline Obesidade & 16,4 & $12,3-20,6$ & 12,2 & $10,3-14,0$ & 13,9 & $12,0-15,7$ \\
\hline \multicolumn{7}{|l|}{ Hábito alimentar } \\
\hline FLV regular & 25,0 & $20,4-29,6$ & 29,4 & $26,8-31,9$ & 42,9 & $40,1-45,7$ \\
\hline FLV recomendado & 13,9 & $10,4-17,3$ & 18,2 & $16,0-20,4$ & 25,4 & $22,8-27,9$ \\
\hline Refrigerantes & 26,6 & $21,6-31,7$ & 31,9 & $28,9-34,8$ & 22,0 & $19,6-24,5$ \\
\hline Carnes com excesso de gordura & 44,3 & $38,9-49,8$ & 39,6 & $36,7-42,5$ & 33,9 & $31,2-36,5$ \\
\hline \multicolumn{7}{|l|}{ Atividade física } \\
\hline Ativo suficiente no tempo livre (lazer) & 16,8 & $12,1-21,6$ & 22,7 & $20,3-25,0$ & 25,3 & $22,8-27,7$ \\
\hline Consumo excessivo de álcool & 25,6 & $20,8-30,3$ & 29,6 & $26,9-32,4$ & 26,1 & $23,9-28,3$ \\
\hline Autoavaliação de estado de saúde ruim & 2,3 & $1,2-3,4$ & 2,0 & $1,2-2,8$ & 1,5 & $0,9-2,1$ \\
\hline \multicolumn{7}{|l|}{ Diagnóstico médico de doenças } \\
\hline Hipertensão arterial & 24,6 & $20,6-28,6$ & 15,1 & $13,3-16,9$ & 21,1 & $18,8-23,5$ \\
\hline Diabetes & 6,3 & $4,7-7,9$ & 3,1 & $2,3-3,8$ & 3,8 & $2,8-4,7$ \\
\hline Dislipidemia & 13,5 & $10,6-16,4$ & 14,4 & $12,7-16,2$ & 22,7 & $20,3-25,2$ \\
\hline Osteoporose & 2,2 & $1,3-3,1$ & 0,6 & $0,3-0,9$ & 1,1 & $0,6-1,6$ \\
\hline
\end{tabular}

* Percentual ponderado para ajustar a distribuição sociodemográfica da amostra Vigitel à distribuição da população adulta da cidade no censo demográfico de 2000.

Fonte: Sistema de Vigilância de Fatores de Risco e Proteção para Doenças Crônicas por Inquérito Telefônico (Vigitel), Ministério da Saúde. 
Tabela 5. Distribuição* (\%) de fatores de risco e proteção para doenças crônicas não transmissíveis em mulheres beneficiárias de planos de saúde nas capitais brasileiras, segundo escolaridade, 2008.

\begin{tabular}{|c|c|c|c|c|c|c|}
\hline \multirow{3}{*}{ Indicadores } & \multicolumn{6}{|c|}{ Anos de estudo } \\
\hline & \multicolumn{2}{|r|}{0 a 8} & \multicolumn{2}{|c|}{9 a 11} & \multicolumn{2}{|c|}{12 ou mais } \\
\hline & $\%$ & (IC95\%) & $\%$ & (IC95\%) & $\%$ & (IC95\%) \\
\hline \multicolumn{7}{|l|}{ Hábito de fumar } \\
\hline Fumantes & 13,1 & $10,4-15,7$ & 8,4 & $6,9-9,9$ & 10,5 & $8,9-12,0$ \\
\hline Consumidores de vinte ou mais cigarros & 3,0 & $1,8-4,3$ & 2,1 & $1,3-2,9$ & 2,4 & $1,7-3,1$ \\
\hline Ex-fumantes & 20,0 & $16,9-23,1$ & 14,4 & $12,8-16,0$ & 15,4 & $13,8-17,0$ \\
\hline \multicolumn{7}{|l|}{ Estado nutricional } \\
\hline Excesso de peso & 51,3 & $46,9-55,8$ & 32,7 & $30,3-35,0$ & 30,5 & $28,4-32,6$ \\
\hline Obesidade & 18,9 & $15,7-22,0$ & 8,1 & $6,9-9,3$ & 8,0 & $6,8-9,3$ \\
\hline \multicolumn{7}{|l|}{ Hábito alimentar } \\
\hline FLV regular & 40,7 & $36,7-44,7$ & 38,9 & $36,5-41,4$ & 51,8 & $49,6-54,1$ \\
\hline FLV recomendado & 25,5 & $21,8-29,3$ & 24,0 & $21,8-26,1$ & 34,6 & $32,4-36,7$ \\
\hline Refrigerantes & 21,1 & $17,3-25,0$ & 22,5 & $20,3-24,8$ & 13,4 & $11,9-15,0$ \\
\hline Carnes com excesso de gordura & 24,2 & $20,5-27,8$ & 22,4 & $20,3-24,4$ & 17,1 & $15,4-18,8$ \\
\hline \multicolumn{7}{|l|}{ Atividade física } \\
\hline Ativo suficiente no tempo livre (lazer) & 11,4 & $9,2-13,7$ & 15,4 & $13,7-17,0$ & 16,3 & $14,7-17,9$ \\
\hline Consumo excessivo de álcool & 8,2 & $5,7-10,7$ & 11,3 & $9,6-13,0$ & 11,7 & $10,2-13,1$ \\
\hline Autoavaliação de estado de saúde ruim & 7,4 & $5,5-9,3$ & 3,2 & $2,4-3,9$ & 1,9 & $1,4-2,5$ \\
\hline \multicolumn{7}{|l|}{ Diagnóstico médico de doenças } \\
\hline Hipertensão arterial & 35,2 & $31,7-38,7$ & 13,7 & $12,4-15,1$ & 14,1 & $12,6-15,6$ \\
\hline Diabetes & 9,0 & $7,4-10,5$ & 3,0 & $2,4-3,6$ & 2,6 & $2,0-3,2$ \\
\hline Dislipidemia & 27,4 & $24,2-30,5$ & 15,7 & $14,1-17,3$ & 17,1 & $15,6-18,6$ \\
\hline Osteoporose & 10,9 & $9,3-12,6$ & 3,5 & $2,9-4,2$ & 3,4 & $2,7-4,1$ \\
\hline \multicolumn{7}{|l|}{ Exames preventivos } \\
\hline Mamografia $^{* *}$ & 80,6 & $76,8-84,4$ & 88,0 & $85,1-90,8$ & 91,9 & $90,0-93,9$ \\
\hline Citologia oncótica $^{* * *}$ & 86,9 & $83,2-90,5$ & 89,0 & $87,2-90,8$ & 93,6 & $92,6-94,7$ \\
\hline
\end{tabular}

"Percentual ponderado para ajustar a distribuição sociodemográfica da amostra Vigitel à distribuição da população adulta da cidade no censo demográfico de 2000; " Exame realizado em mulheres de 50 a 59 anos nos últimos dois anos; "** Exame realizado em mulheres de 25 a 59 anos nos últimos três anos.

Fonte: Sistema de Vigilância de Fatores de Risco e Proteção para Doenças Crônicas por Inquérito Telefônico (Vigitel), Ministério da Saúde.

nas $2,0 \%$ das pessoas em domicílios com rendimento per capita $<0,25$ salário mínimo possuíam plano de saúde versus $63,2 \%$ daqueles com rendimento $>5$ salários mínimos ${ }^{8}$.

Como os dados do IBGE não estão disponíveis para as capitais, para fins de comparação foram utilizadas informações cadastrais da Agência Nacional de Saúde Suplementar, que referem cerca de $40 \%$ da população coberta por planos de saúde ${ }^{11}$, valor bastante próximo ao deste estudo $(41,8 \%)$, que não excluiu beneficiários de planos públicos (municipal, estadual, militar).

Nos últimos anos tem sido dada ênfase a ações de promoção e prevenção das DCNT tanto por parte da Agencia Nacional de Saúde Suplementar $^{12}$ quanto pelas operadoras de planos de saúde no Brasil ${ }^{13}$ e em outros países ${ }^{14}$. O estudo atual contribui para o aprofundamento dessas ações.

$\mathrm{O}$ estudo atual ainda possibilita monitorarse um conjunto de FR, dentre eles o tabagismo, que é considerado pela OMS como a principal causa de morte evitável. O tabagismo constituise também como um dos principais fatores de risco para diversas doenças, como as doenças cardiovasculares, as neoplasias e as doenças respiratórias obstrutivas crônicas ${ }^{15}$. O estudo atual mostra $14,0 \%$ de fumantes, sendo que o IBGE apontou prevalências maiores de tabagismo na população brasileira acima de 15 anos $(17,2 \%)^{16}$, o que pode ser explicado pela maior escolaridade e renda da população coberta por planos, o que 
é compatível com outros estudos que mostram a redução da prevalência do tabagismo entre aqueles com maior escolaridade e com aumento da maior idade ${ }^{15,16}$.

A prevalência de excesso de peso e obesidade aumentou nas últimas décadas em praticamente todos os países do mundo, inclusive no Brasil ${ }^{17}$. A mudança se deve ao desenvolvimento econômico mundial levando a alterações nos hábitos e estilos de vida, com consumo excessivo de alimentos ricos em gordura saturada associado aos baixos níveis de atividade física, o que aumenta o risco de doenças cardíacas e cerebrovasculares, hipertensão, diabetes, câncer e desordens musculoesqueléticas ${ }^{1,2,4}$.

O estudo atual mostrou prevalências maiores de sobrepeso e obesidade comparado com a Pesquisa de Orçamentos Familiares (POF) de 2003, que mostrou sobrepeso em $41,1 \%$ dos homens e $40,0 \%$ das mulheres ${ }^{18}$. Entretanto, comparado com a população de planos de uma operadora de saúde americana, a obesidade foi menor ${ }^{19}$.

O padrão alimentar dos indivíduos é um importante condicionante da morbimortalidade por doenças crônicas ao longo de todas as fases da vida. De acordo com a OMS, o consumo insuficiente de frutas, legumes e verduras (consumo diário inferior a 400 gramas ou, aproximadamente, cinco porções por pessoa) é responsável anualmente por 2,7 milhões de mortes e por $31 \%$ das doenças isquêmicas do coração, $11 \%$ das doenças cerebrovasculares e $19 \%$ dos cânceres gastrointestinais ocorridos em todo o mundo ${ }^{1,20}$. Ainda segundo a OMS, as dislipidemias, causadas majoritariamente pelo consumo excessivo de gorduras saturadas de origem animal, também aumentam as doenças cerebrovasculares e doenças isquêmicas do coração ${ }^{20}$. Assim, a OMS preconizou na Estratégia Global para Alimentação, Atividade Física e Saúde a adoção de hábitos saudáveis, incluindo o aumento do consumo de frutas, legumes e verduras ${ }^{20}$.

O estudo atual, de forma semelhante ao padrão ocidental, mostrou que a população beneficiária de planos não tem alimentação adequada, apenas $22 \%$ ingerem o recomendado de FLV, quase um terço ingere carnes com gorduras e um quarto refrigerantes.

Estudos epidemiológicos demonstram associação entre estilo de vida ativo e melhor qualidade de vida. A atividade física e o exercício previnem efetivamente a ocorrência de eventos cardíacos, reduzem a incidência de acidente vascular cerebral, hipertensão, diabetes mellitus do tipo 2, cânceres de cólon e mama, fraturas osteopo- róticas, doença vesicular, obesidade, depressão e ansiedade, além de retardarem a mortalidade ${ }^{21}$. Essas recomendações são seguidas apenas por $17,6 \%$ da população que têm planos de saúde.

As maiores frequências de excesso de peso e obesidade nas maiores idades e menores frequências entre mulheres com maior escolaridade são compatíveis com outros estudos, mostrando que as mulheres mais escolarizadas são mais cuidadosas, praticam mais atividade física e alimentação saudável ${ }^{22}$. Já o aumento dessas condições com a idade pode ocorrer em razão de hábitos mais sedentários, maior ingestão de alimentos calóricos e/ou fatores genéticos e metabólicos.

O consumo abusivo de bebidas alcoólicas pode levar a doenças como cirrose hepática, pancreatite crônica, insuficiência cardíaca, acidente vascular cerebral, neoplasias, além de ocorrências como acidentes de trânsito e violência, dentre outras $^{23,24}$.

Os dados do estudo atual mostram 17,6\% da população com consumo abusivo de bebidas alcoólicas, sendo maior entre homens e jovens. Este padrão é consistente com outros estudos nacionais e internacionais ${ }^{23,24}$, chamando a atenção para o risco da exposição de álcool, direção e acidentes de trânsito.

A autoavaliação do estado de saúde vem sendo utilizada em vários estudos ${ }^{8,25}$, sendo considerada um método confiável, capaz de expressar vários aspectos da saúde física, cognitiva e emocional dos indivíduos. A autoavaliação do estado de saúde mostrou ser um importante indicador de mortalidade: pessoas com pior percepção do estado de saúde têm maior risco de morte (por todas as causas) em comparação com as que relatam saúde excelente ${ }^{25}$.

O estudo atual mostrou que os idosos avaliam pior seu estado de saúde, bem como a população com escolaridade menor. Estudos confirmam que os idosos avaliam pior sua saúde, pois este indicador está relacionado ao declínio funcional, sendo utilizado em pesquisas gerontológi$\mathrm{cas}^{25}$. A PNAD também mostrou pior avaliação do estado de saúde entre mulheres e indivíduos com menor escolaridade, renda e entre idosos ${ }^{8}$.

O câncer é responsável por mais de $12 \%$ de todas as causas de óbito no mundo, com cerca de dez milhões de casos novos e sete milhões de mortes por $a^{26}{ }^{26}$. Evidências mostram que hábitos alimentares inadequados e a falta de atividade física têm um papel distinto como fator de risco para o câncer. Além disso, o consumo excessivo de álcool é responsável pela ocorrência de diversos tipos de câncer como os de esôfago, fa- 
ringe, laringe, fígado e mama, por exemplo ${ }^{26}$. Segundo estimativas da OMS, cerca de $40 \%$ dos casos de câncer poderiam ser evitados por meio de medidas preventivas como dieta saudável, atividade física regular e ausência do tabaco ${ }^{1,3,26}$.

O exame mamográfico é uma das principais medidas preventivas para detecção precoce do câncer de mama. No Brasil, o Ministério da Saúde recomenda como principais estratégias de rastreamento populacional um exame mamográfico, pelo menos a cada dois anos, para mulheres de 50 a 69 anos de idade ${ }^{3,27}$. Dados da Pesquisa Nacional por Amostra de Domicílios (PNAD) levantados pelo Instituto Brasileiro de Geografia e Estatística em 2008 mostraram que, em relação à mamografia, $71,1 \%$ das mulheres de 50 anos ou mais referiram já ter se submetido a um exame alguma vez em suas vidas, crescendo para 95,6\% entre aquelas de maior renda ${ }^{8}$. O estudo atual foi compatível com os dados da PNAD, mostrando que a população beneficiária de planos apresentou coberturas mais elevadas; $83,6 \%$ das mulheres com mais de 50 anos realizaram mamografia nos últimos dois anos.

O Ministério da Saúde preconiza que todas as mulheres entre 25 e 59 anos ou as mais jovens com vida sexual ativa realizem exame de colo do útero a cada três anos, sendo recomendada a realização de exames anuais para mulheres com citologia alterada ${ }^{27}$. O estudo atual mostrou coberturas mais elevadas $(88,7 \%)$ do que a da população geral $(83,2 \%)$. Esta diferença é compatível com os dados da PNAD que mostraram cobertura de $87,1 \%$ na população geral e $96,2 \%$ na população com renda acima de cinco salários mínimos. A maior cobertura na população de planos de saúde denota a maior facilidade de acesso aos serviços de saúde dessa população já identificada pela PNAD $2008^{8}$.

Dados da PNAD mostram maior prevalência de doenças crônicas nas maiores idades e menor prevalência nas maiores escolaridade e renda ${ }^{8}$.

Os estudos populacionais sobre hipertensão, diabetes e outras doenças crônicas são regionalizados, dificultando conclusões sobre prevalências globais. Estudo sobre prevalência de hipertensão arterial encontrou valores próximos a $20 \%{ }^{28}$. Os achados do atual estudo são semelhantes: $23,4 \%(21,9 \%-24,8 \%)$ de prevalência na população de planos, maior prevalência entre mulheres, idades avançadas e baixa escolaridade. A maior prevalência entre mulheres pode ser explicada pela maior procura dos serviços de saúde pelas mulheres e, portanto, maior oportunidade de acesso. O aumento nas populações idosas deve-se ao envelhecimento e aumento das comorbidades. Quanto à população com maior escolaridade, o acesso às medidas de promoção e prevenção poderia explicar a menor prevalência de doenças.

Estudo multicêntrico realizado em nove capitais brasileiras em 1998 mostrou prevalência média de diabetes mellitus de 7,6\% em indivíduos com mais de 30 anos, variando de $3 \%$ na faixa etária de 30 a 39 anos até $17 \%$ naqueles entre 60 e 69 anos de idade ${ }^{29}$. O estudo atual mostra prevalência de 5,6\% em maiores de 18 anos, maior nas maiores idades, o que é compatível com a literatura $^{8,29}$.

A osteoporose é uma desordem esquelética que atinge principalmente as mulheres acima de 50 anos de idade, sendo responsável pelo aumento do risco de fraturas ${ }^{30}$. Dados da América Latina apontam $12 \%$ a $18 \%$ de osteoporose em coluna vertebral e de $8 \%$ a $22 \%$ em fêmur proximal entre as mulheres com idade igual ou superior a 50 $\operatorname{anos}^{31}$. Todavia, pouco se sabe sobre a sua prevalência na população brasileira. Estudo desenvolvido em São Paulo com 301 idosos com 70 ou mais anos de idade mostrou $22 \%$ a $33 \%$ de osteoporose entre as mulheres e $6 \%$ a $16 \%$ entre os homens $^{32}$. O estudo atual encontrou $4,7 \%$, sendo maior nas mais altas idades entre mulheres de menor escolaridade, conforme literatura ${ }^{33}$.

Os limites do estudo devem-se ao fato de a amostra restringir-se àqueles que possuem telefone fixo, com menores coberturas nas regiões Norte e Nordeste. Todavia, o uso de fatores de ponderação reduz esses viéses, buscando aproximar-se da população do censo de 2000. Além disto, o recorte para a população beneficiária de planos reduziu a população estudada para $40 \%$ da amostra inicial. Ainda assim, a amostra é suficiente para definir as estimativas com precisão para o conjunto das localidades avaliadas.

As informações contidas no atual trabalho constituem dados sobre o perfil dos fatores de risco e proteção para DCNT no setor de saúde suplementar, facilitando o planejamento e o monitoramento de ações de promoção da saúde e prevenção de riscos e doenças. 


\section{Colaboradores}

DC Malta trabalhou na concepção e no delineamento do estudo, na análise e interpretação dos dados, redigiu a primeira versão do artigo, trabalhou na sua revisão crítica e aprovou a versão a ser publicada; MR Oliveira, EC Moura, SA Silva, FP Santos e CS Zouain trabalharam na concepção e no delineamento do estudo, na análise e interpretação dos dados, na sua revisão crítica e aprovaram a versão a ser publicada; OL Morais Neto e GO Penna trabalharam na concepção e no delineamento do estudo, na sua revisão crítica e aprovaram a versão a ser publicada.

\section{Agradecimentos}

Os autores agradecem a equipe técnica da ANS, Danielle Conte Alves, Jacqueline Alves Torres, Jorge Luizi Pinho, José Felipe Riani Costa, Kátia Audi Curci, Michelle Mello de Souza Rangel e Renata Fernandes Cachapuz, que colaboraram na revisão de literatura do inquérito Vigitel Brasil 2008: Saúde Suplementar, base para o atual trabalho.

\section{Referências}

1. Organização Mundial da Saúde. Prevenção de doenças crônicas: um investimento vital. Genebra: OMS; 2005.

2. Brasil. Ministério da Saúde. Diretrizes e recomendações para o cuidado integral de doenças crônicas não transmissíveis: promoção da saúde, vigilância, prevenção e assistência. Brasília: Ministério da Saúde; 2008.

3. Brasil. Instituto Nacional do Câncer. Estimativa 2006: incidência de câncer no Brasil. Rio de Janeiro: Inca; 2005.

4. Malta DC, Cezário AC, Moura L, Morais Neto OL, Silva Júnior JB. Construção da vigilância e prevenção das doenças crônicas não trasmissíveis no contexto do Sistema Único de Saúde. Epidemiol Serv Saúde 2006; 15:47-64.

5. Passos VMA, Assis TD, Barreto SM. Hipertensão arterial no Brasil: estimativa de prevalência a partir de estudos de base populacional. Epidemiol Serv Saúde 2006; 15:35-45.

6. Brasil. Ministério da Saúde. Secretaria de Vigilância em Saúde. Vigitel Brasil 2007: vigilância de fatores de risco e proteção para doenças crônicas por inquérito telefônico. Brasília: Ministério da Saúde; 2007.

7. Moura EC, Morais Neto OL, Malta DC, Moura L, Silva NN, Bernal R, Claro RM, Monteiro CA. Vigilância de fatores de risco para doenças crônicas por inquérito telefônico nas capitais dos 26 estados brasileiros e no Distrito Federal (2006). Rev Bras Epidemiol 2008; 11:20-37.

8. Instituto Brasileiro de Geografia e Estatística (IBGE). Pesquisa Nacional por Amostra de Domicílios 2008. Suplemento Saúde. Brasil: IBGE; 2010.

9. Brasil. Ministério da Saúde. Vigitel Saúde Suplementar: vigilância de fatores de risco e proteção para doenças crônicas por inquérito telefônico. Brasília, Rio de Janeiro: Agência Nacional de Saúde Suplementar, Ministério da Saúde; 2009.

10. Stata. Stata Statistical Software: Release 9.0. Texas: Stata Corporation; 2005.

11. Agência Nacional de Saúde Suplementar. Caderno de informações. Rio de Janeiro: ANS; 2009. [acessado 2009 mar]. Disponível em: http://www.ans.gov.br/ data/files/8A9588652AC9E68D012B1C5C570D4E82/ Caderno_2010-09-WEB.pdf

12. Agência Nacional de Saúde Suplementar. Promoção da saúde e prevenção de riscos e doenças na saúde suplementar: manual técnico. $2^{\mathrm{a}}$ ed. rev. e atual. Rio de Janeiro: ANS; 2007.

13. Malta DC, Jorge AO. Modelos assistenciais na saúde suplementar: o caso de uma operadora de autogestão. Cien Saude Colet [periódico na Internet] 2008; 13(5):1535-1542. [acessado 2009 mar 01]. Disponível em: http://www.scielo.br/scielo.php?script=sci_ arttext\&pid=S1413-81232008000500018\&lng=pt

14. Porter ME. Repensando a saúde: estratégias para melhorar a qualidade e reduzir os custos. Porto Alegre: Bookman; 2007.

15. Malta DC, Moura EC, Silva SA, Oliveira PP, Costa e Silva VL. Prevalência do tabagismo em adultos residentes nas capitais dos estados e no Distrito Federal, Brasil 2008. J Bras Pneumol 2010; 36(1):75-83. 
16. Instituto Brasileiro de Geografia e Estatística. Pesquisa Nacional do Tabagismo - Petab. Suplemento Saúde da Pesquisa Nacional por Amostra de Domicílios 2008. Brasil: IBGE; 2009.

17. Stein CJ, Colditz GA. The epidemic of obesity. J Clin Endocrinol Metab 2004; 89:2522-2525.

18. Instituto Brasileiro de Geografia e Estatística. Pesquisa de Orçamentos Familiares 2002-2003: análise da disponibilidade domiciliar de alimentos e do estado nutricional no Brasil. Brasil: IBGE; 2004.

19. Frolich A, Schiotz ML, Larsen-Strandberg M, Hsu J, Krasnik A, Diderichsen F, Bellows J, Sogaard J, White $\mathrm{K}$. A retrospective analysis of health systems in Denmark and Kaiser Permanente. BMC Health Services Research 2008; 8:1-8. [acessado 2009 jun 16]. Disponível em: http://www.biomedcentral.com/ content/pdf/1472-6963-8-252.pdf

20. World Health Organization. Global strategy on diet, physical activity and health. Geneva: WHO; 2004.

21. ACSM. American College of Sports Medicine. Diretrizes do ACSM para os testes de esforço e sua prescrição. Traduzido por Giuseppe Taranto. 7a. ed. Rio de Janeiro: Guanabara Koogan, 2007.

22. Matheson FI, Moineddin R, Glazier RH. The weight of place: A multilevel analysis of gender, neighborhood material deprivation, and body mass index among Canadian adults. Soc Sci Med. 2008; 66:67590.

23. World Health Organization. International guide for monitoring alcohol consumption and related harm. Geneva: WHO; 2000.

24. Mascarenhas MDM, Malta DC, Silva MMA, Carvalho CG, Monteiro RA, Morais Neto OL. Consumo de álcool entre vítimas de acidentes e violências atendidas em serviços de emergência no Brasil, 2006 e 2007. Cien Saude Colet 2009; 14(5):1789-1796.

25. Alves LS, Rodrigues RN. Determinantes da autopercepção de saúde entre idosos do município de São Paulo, Brasil. Rev Panam Salud Publica 2005; 17:333-341. [acessado 2009 jul 14]. Disponível em: http://www.scielosp.org/scielo.php?pid=S1020 49892005000500005\&script $=$ sci_arttext

26. World Health Organization. Cancer. Geneva: WHO; 2009. [acesso 2009 jun 17]. Disponível em: http:// www.who.int/mediacentre/factsheets/fs297/en/index. html
27. Instituto Nacional do Câncer. Programa Nacional de Controle do Câncer de Colo do Útero e de Mama Viva Mulher. [acessado 2010 abr 7]. Disponível em: http//www.inca.gov.br/conteúdo_view.asp?id=140

28. Piccini RX, Victora CG. Hipertensão arterial sistêmica em área urbana no sul do Brasil: prevalência e fatores de risco. Rev Saude Publica 1994; 28(4):261267.

29. Schmidt MI. Diabete melito: diagnóstico, classificação e abordagem inicial. In: Duncan BB, Schmidt MI, Giugliani ERJ. Medicina ambulatorial: condutas de atenção primária baseadas em evidências. $3^{\mathrm{a}}$ ed. Porto Alegre: Artmed; 2006.

30. National Institute of Health (NIH) Consensus Statement. Osteoporosis prevention, diagnosis and therapy. JAMA 2001; 285:785-795.

31. Reid IR. Relationships among body mass, its components, and bone. Bone 2002; 31:547-555.

32. Camargo MB, Cendoroglo MS, Ramos LR, Oliveira Latorre MR, Saraiva GL, Lage A, Carvalhaes Neto $\mathrm{N}$, Araújo LM. Bone mineral density and osteoporosis among predominantly Caucasian elderly population in the city of São Paulo, Brazil. Osteoporos Int 2005; 16(11):1451-1460.

33. Martini LA, Moura EC, Santos LC, Malta DC, Pinheiro MM. Prevalência de diagnóstico auto-referido de osteoporose, Brasil, 2006. Rev Saude Publica [site da Internet]. [acessado 2010 jul] Disponível em: http://www.scielosp.org/scielo.php?script=sci_arttext \&pid=S0034-89102009000900014\&lng=en

Artigo apresentado em 30/05/2010

Aprovado em 08/07/2010

Versão final apresentada em 15/07/2010 